\section{P58 (continued)}

Outcome Measures and Analysis: Paired t-tests were used to measure changes in the total number of FV that (1) children have tried and (2) they "like a little"/"like a lot" from baseline to post-intervention.

Results: Children reported an increased number of FV tried (mean increase 2.3, $\mathrm{t}=5.88, \mathrm{p}<0.001$ ) and an increased number in the FV liked (mean increase 2.1, $\mathrm{t}=3.45, \mathrm{p}=0.009$ ) post-intervention. Families reported "trying new foods" as a program highlight and tastetesting was an important component in helping families eat more healthfully.

Conclusions and Implications: This simple, low cost activity allowed children the opportunity to try new FV and increase FV exposure and intake. Educators and researchers are encouraged to consider this taste-testing activity to promote FV intake and support positive behavior change in children.

Funding: NIH.

\section{P59 Interviews Inform Translation of In-School Intervention for Out-of-School Settings: Staff, Student and Curricular Challenges}

Divyani Sarkar, MS, RD, Divyani.Sarkar@Colostate.edu, Colorado State University, 1571 Campus Delivery, Fort Collins, CO 80521; B. Lohse, PhD, RD, Pennsylvania State University; A. Cutler, Colorado State University;

L. Cunningham-Sabo, PhD, RD

Objective: To determine potential opportunities and constraints of adapting Fuel for Fun (FFF), an in-school integrated research, education and extension project that experientially promotes healthy eating and activity, to an out-of-school setting.

Design, Setting and Participants: Eleven out-ofschool program coordinators were recruited using purposive sampling for 1-time interviews. These programs address nutrition and childhood obesity and represented variety in location and participant demographics with potential for FFF adaptation partnership.

Outcome Measures and Analysis: Individual semistructured telephone interviews were audio-recorded, transcribed and systematically coded by two researchers using an inductive approach and QSR NVivo. Coded statements were gathered into categories and sub-categories, and then collapsed into themes to identify key concepts. Inter-coder agreement of $>95 \%$ was achieved.

Results: Each interview was completed in approximately 30 minutes. Main themes that emerged included high staff turnover and challenges with retaining participants, making training and consistent program delivery a challenge, Food expense when implementing a cooking and/or nutrition program were also mentioned. Curricular needs included lessons with readily available ingredients for rural communities and adaptability to a wide age-range of participants. Respondents reported scheduling conflicts and lack of interest challenged family involvement. Sugges- tions to enhance family participation were recruiting family as volunteers, conducting cooking classes with parents and children, and having an end-of-program celebratory activity.

Conclusions and Implications: Results confirmed the importance of curricular adaptability for a wider age-range and adequate funding. Building relationships with organizations that can sustain the program and provide on-going training for a successful out-of-school program is also critical.

Funding: NIFA.

\section{P60 Measurements of the Built Environment for Nutrition and Physical Activity in Rural Low-Income Ethnic Communities in Kansas}

Yijing Li, MS, yijing@ksu.edu, Kansas State University, Manhattan, KS 66506; T. Kidd, PhD, RD, LPN;

E. Lindshield, MPH, RD, LD; K. Adhikari, PhD;

N. Muturi, PhD; K. Kattelmann, PhD, RD, LN, South Dakota

State University; S. Zies, MEd, Ohio State University

Objective: Nutrition and physical activity (PA) have been highlighted as important factors in disease prevention and health promotion, which can be largely affected by the built environment. As part of a 5-year multi-state adolescent obesity prevention project, the built environment for nutrition and PA were assessed in rural low-income ethnic communities in Kansas.

Design, Setting and Participants: One control and one intervention communities in Southwest Kansas were randomly selected prior to program development.

Outcome Measures and Analysis: In-person audits of restaurants ( $\mathrm{n} 1=9, \mathrm{n} 2=3$; respectively for the control and intervention communities) and stores ( $\mathrm{n} 1=7, \mathrm{n} 2=$ $2)$, as well as street segments (n1 = 3, n2 =5) and parks ( $\mathrm{n} 1=4, \mathrm{n} 2=2$ ) were conducted by trained observers using validated Nutrition Environment Measures Survey-Restaurant and Store (NEMS-R and NEMS-S), Active Neighborhood Checklist (ANC), and Physical Activity Resource Assessment (PARA) to document and describe current neighborhood conditions.

Results: Nutrition information and healthier options were provided at $11.1 \%$ and $33.3 \%$ of the restaurants, respectively. The availability of fruits and vegetables, however, varied by stores. The majority PA structures available were play sets $(72.5 \%)$ and basketball courts (51.0\%). Sidewalks (78.5\%) and stop signs (69.0\%) were present to promote neighborhood safety for walking and cycling.

Conclusions and Implications: The built environments for nutrition and PA in both the control and intervention communities were similar, which suggests future studies on the perceived measures of the built environments are required to understand how improvements made in the intervention communities could increase the overall well-being of adolescents.

Funding: NIFA. 